\title{
Primary Site of Disease
}

National Cancer Institute

\section{Source}

National Cancer Institute. Primary Site of Disease. NCI Thesaurus. Code C158874.

The primary site of a disease of interest. 